\title{
Signal Processing Techniques for the Characterization Ultrasound Complex Materials
}

\author{
A. Khireddine ${ }^{(1)}$, J.P Salvestrini ${ }^{(2)}$
}

\begin{abstract}
This article focuses on the application of different signal processing techniques to improve the visibility of the echoes during travel time between the echo of entry (opposite echo), and the back wall echo (back surface) of longitudinal and transverse waves, it is easy to calculate the speed and longitudinal cross respectively, and then determine the elastic constants (Young modulus, Poisson's ratio,) that allow us to describe the development of properties andsplit spectrum processing method.
\end{abstract}

Index Terms- Echoes, Ultra sound, Split spectrum
processing, transformation

\section{INTRODUCTION}

During the production of certain products made of raw materials, several defects can penetrate inside the material (grain, crack,). So it is necessary to know the characteristics of the materials and identify the presence of defects and to determine their nature and their positions.

It is within this framework that the work we have developed to implement the various techniques of signal processing to improve the signal to noise ratio (Split spectrum processing, wavelet transformation), which then allows us to measure the delay different echoes.

\section{Split Spectrum Processing}

In the late 70 's, a technique called Split Spectrum Processing was developed for the implementation of the concept of diversity frequency (frequency diversity), to improve the signal noise in order to process signals from granular materials such as concrete [1-3].

A whole range of frequencies is created from a single input signal to broadband, using a number of parallel band filters Gaussian shape as shown in Figure 1. The resulting spectra were processed in time domain using the inverse Fourier transform, they are then multiplied by factors $\mathrm{W} 1, \mathrm{WN}$. These factors are chosen such that the amplitude of each signal band is close to a standard unit $\mathrm{N}$ narrowband signals $\mathrm{W} 1 \mathrm{X} 1 \mathrm{(t)}$.... WNXN $(\mathrm{t})$ are processed and recombined using several linear and nonlinear for an output

Manuscript received October 9, 2009

A. Khireddine is with the Electronic department, Faculty of sciences, University of Bejaia, Algeria. abdelkrim khireddine@hotmail.com).

J.P Salvestrini is with the LMOPS laboratory, University of Metz, France. (e-mail: J.P Salvestrini @univ-metz.fr).

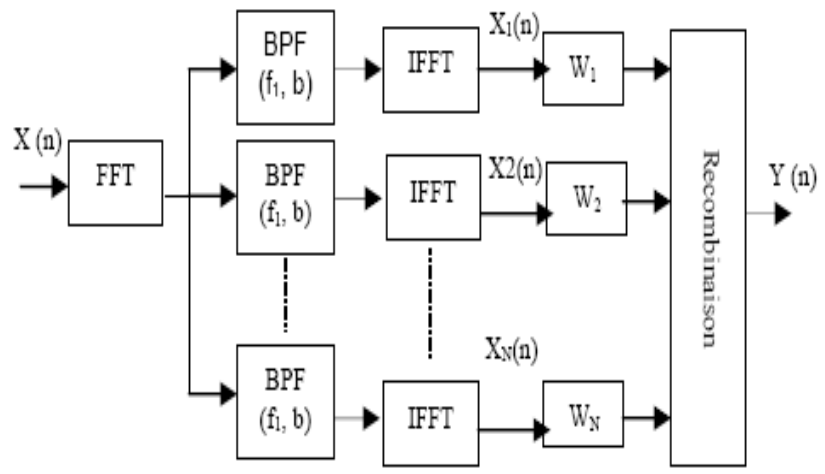

Figure 1. Patterns art of Split Spectrum Processing

Among the important characteristics of the filterand recombination algorithm, we can cite:

-Minimisation

$\mathrm{Y}(\mathrm{n})=\min \left(\left|\mathrm{X}_{1}(\mathrm{n})\right|,\left|\mathrm{X}_{2}(\mathrm{n})\right|,\left|\mathrm{X}_{3}\right| \ldots\left|\mathrm{X}_{\mathrm{n}}(\mathrm{n})\right|\right)$

- Maximisation

$\mathrm{Y}(\mathrm{n})=\max \left(\left|\mathrm{X}_{1}(\mathrm{n})\right|,\left|\mathrm{X}_{2}(\mathrm{n})\right|,\left|\mathrm{X}_{3}(\mathrm{n})\right| \ldots\left|\mathrm{X}_{\mathrm{n}}(\mathrm{n})\right|\right)$

- Medium

- Geométrical mean

$\mathrm{Y}(\mathrm{n})=\left(\left|\mathrm{X}_{1}(\mathrm{n})\right|,\left|\mathrm{X}_{2}(\mathrm{n})\right|,\left|\mathrm{X}_{3}(\mathrm{n})\right| \ldots\left|\mathrm{X}_{\mathrm{n}}(\mathrm{n})\right|\right)^{1 / \mathrm{N}}$

\section{WAVELET TRANSFORM}

Wavelet transform can be defined as the projection on the basis of wavelet functions. The wavelet coefficients are defined as follows:

$$
\begin{gathered}
T O(a, b)=\frac{1}{\sqrt{a}} \int_{-\infty}^{+\infty} f(t) \psi\left(\frac{t-b}{a}\right) d t \\
\text { avec } a, b \in \Re \quad a \neq 0 \\
T O(a, b)=\int_{-\infty}^{+\infty} f(t) \psi_{a, b} d t \\
\text { avec } \psi_{a, b}(t)=\frac{1}{\sqrt{a}} \psi\left(\frac{t-b}{a}\right)
\end{gathered}
$$

The wavelet coefficients (TO $(\mathrm{a}, \mathrm{b})$ ) depends on two parameters $a$ and $b$, where $a$ is the scale factor and $b$ the translation factor. The no translation at $\mathrm{a}$ is $\mathrm{b} / \mathrm{a}$.

The functions are obtained from the expansion and the translation of the mother wavelet function. The are sometimes called wavelet girls.

The scalogramme is a time-scale (time on the horizontal axis, the scale on the vertical axis and the value of gray level of color range).

The scalogramme is expressed from the continuous wavelet transformation. It is defined as the square of the module of the wavelet transformation.

$$
|T O(a, b)|^{2}=\left|a^{\frac{-1}{2}} \int_{-a}^{a} f(t) \psi\left(\frac{t-b}{a}\right) d t\right|^{2}
$$


The wavelet transformation is a multi-resolution representation of a signal. It has become a very powerful tool for non-destructive testing and monitoring by ultrasound. It allows you to filter the signal to detect and characterize the types of defects (planar or volumetric) of materials and improve the signal to noise [4-5].

\section{APPLICATIONS OF CORRELATION FUNCTIONS}

It defines the function of cross-signals of $2 x(t)$ and $y(t)$ finite energy by:

$$
R_{x y}(\tau)=\int_{-\infty}^{+\infty} x(t) y^{*}(t-\tau) d t
$$

The applications of correlation functions are very high by achieving practical results. Among the applications of essential intercorrelations is comparing and measuring the gap between two signals $x \quad(t)$ and $y(t)$ delayed. This delay is the abscissa of the maximum of the cross, the two signals observed [6].

\section{Hilbert Transformation}

This technique uses the properties of the Hilbert transform of a signal to determine the time gap between two signals. It is studying the Hilbert transform of the impulse response of the system [7].

The four signals (Figure 3, 4, 5 and 6) show the results obtained by the technique of prism on samples from a cube of departure $(45 \times 45 \times 45 \mathrm{~mm} 3)$, and using a transducer center frequency of $1 \mathrm{MHz}$.

a-Echo of the front:

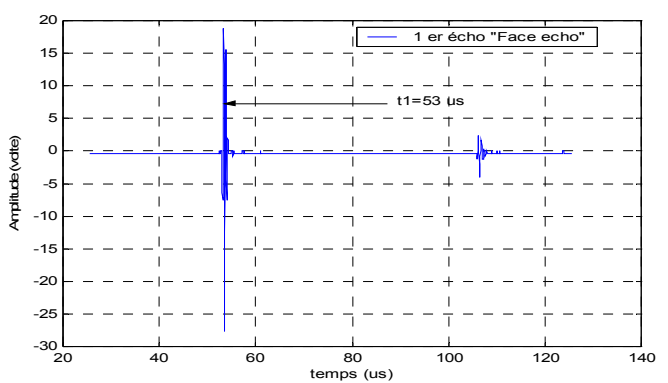

Figure 2. Echo of the front of the sample

Q1: Is the flight time in the water with the sample in his place (normal reflection of the front of the sample).

b-Echo reflector

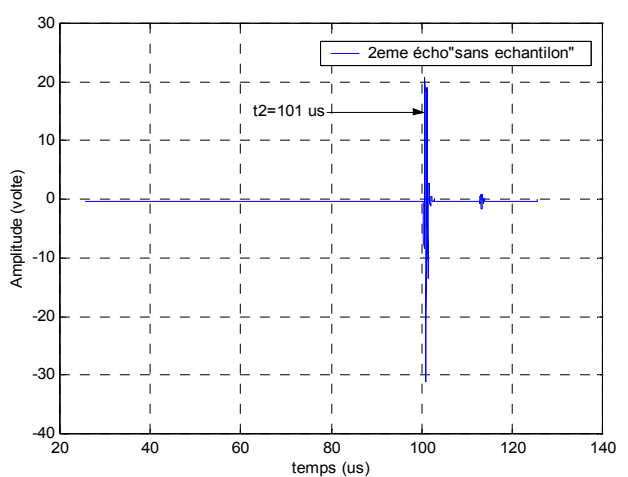

Figure 3. Echo reflector

Q2: Is the flight time in the water sample (normal reflection of the reflector)

c-Echo on longitudinal waves:

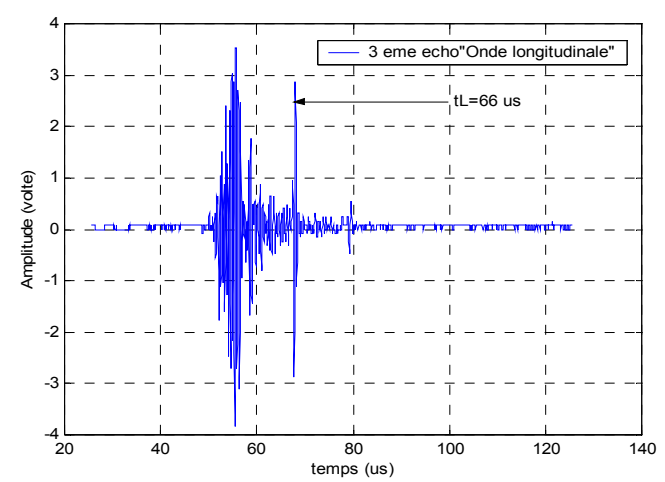

Figure 4. Longitudinal waves

If we increase the angle of attack (by turning around the translator of the sample), it appears a third echo, representing the longitudinal waves.

tL: is the flight time with the presence of the sample, where the refraction occurs at an angle of $45^{\circ}$ within the sample.

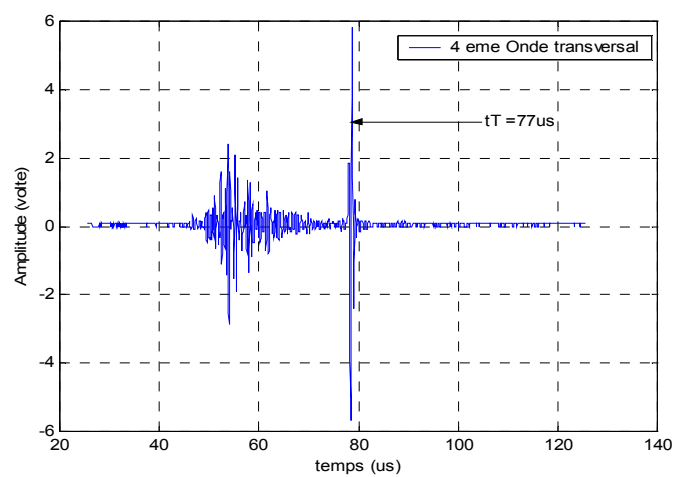

Figure 5. Transverse wave

d-Echo transverse waves :

If we increase further the angle of impact, the third echo disappears (longitudinal waves), and there will appear a fourth echo representing the transverse waves. $\mathrm{tT}$ : is the flight time with the presence of the sample and when refraction occurs at an angle of $35^{\circ}$ within the sample for a transverse wave.

\section{RESUlTS AND DISCUSSION}

The echo of the front (Figure 11) is transformed to frequency domain using the fast Fourier transform. The spectrum is filtered through four filters Gaussians same bandwidth $\mathrm{b}=0.29 \mathrm{MHz}$, and different center frequencies $5 \mathrm{MHz}, 10 \mathrm{MHz}, 15 \mathrm{MHz}$ and $20 \mathrm{MHz}$, with an intersection of $-6 \mathrm{~dB}(50 \%$ overlap, resulting in four signals filtered. filtered signals are processed and recombined using the algorithm recombination (geometric mean) in the range of $0-25 \mathrm{MHz}$ frequency, using increments of frequency $f=10 \mathrm{KHz}$ for the exit as a function of time (Figure 8). 


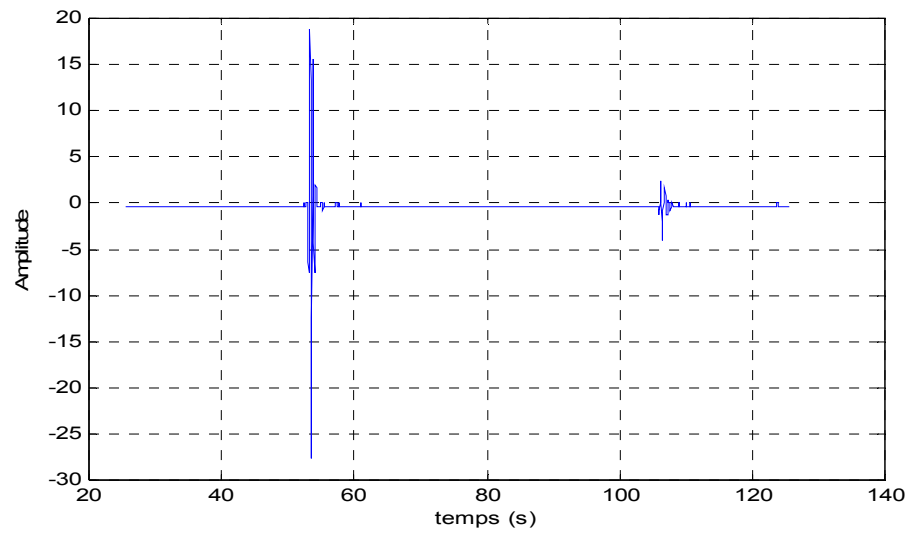

Figure 7. Echo of the front

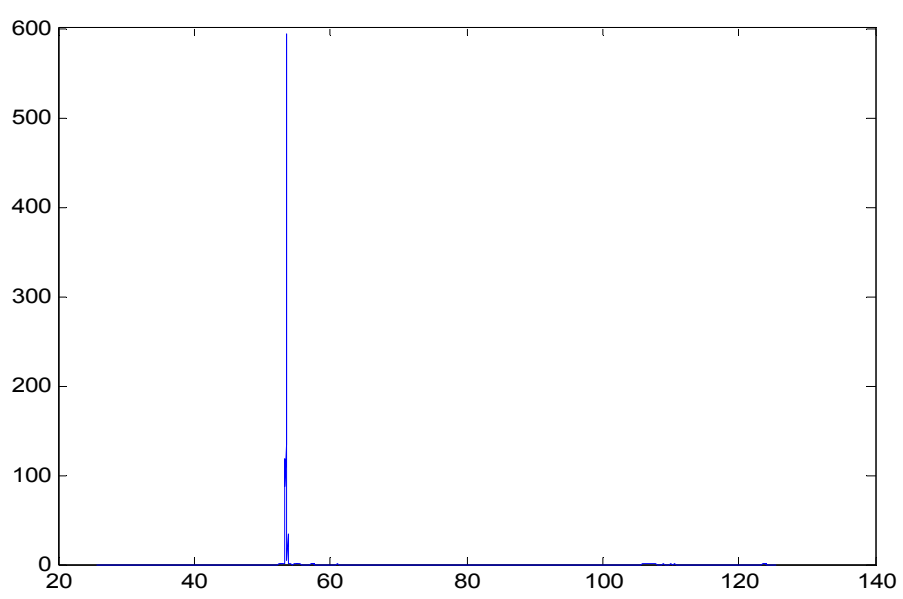

Figure 8. The results of the technical SSP (Geometric mean)

We will apply the same method on the echoes on longitudinal waves (Figure 9) and transverse (Fig. 10), using the same filters and the same algorithms recombination, with the same parameters.

The results of this technique are illustrated in Figures 12,13 .

b-Echo on longitudinal waves

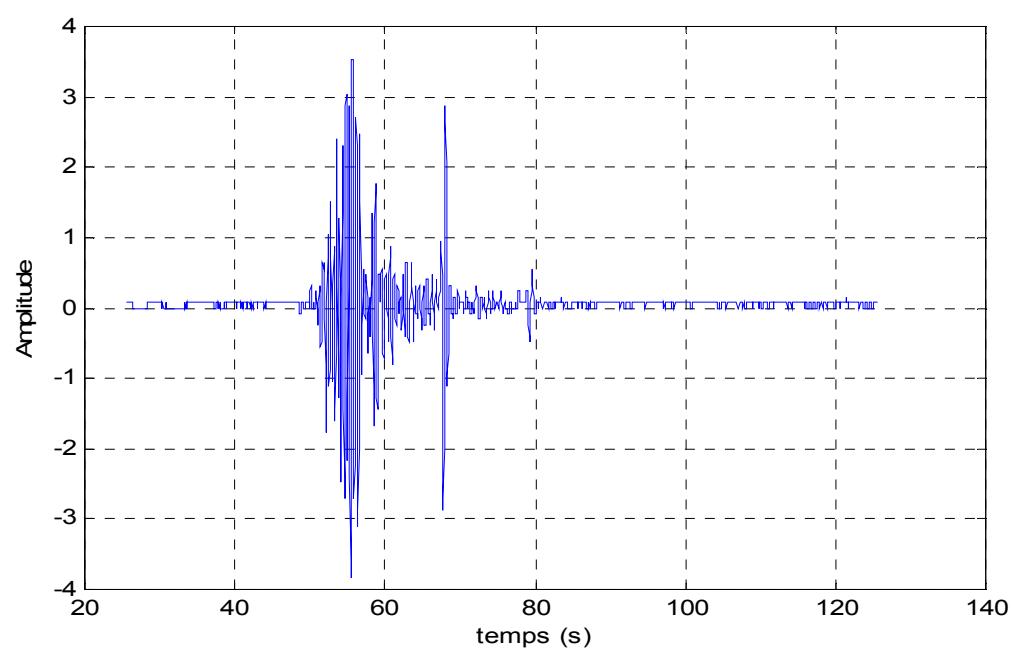

Figure 9. Transverse waves 


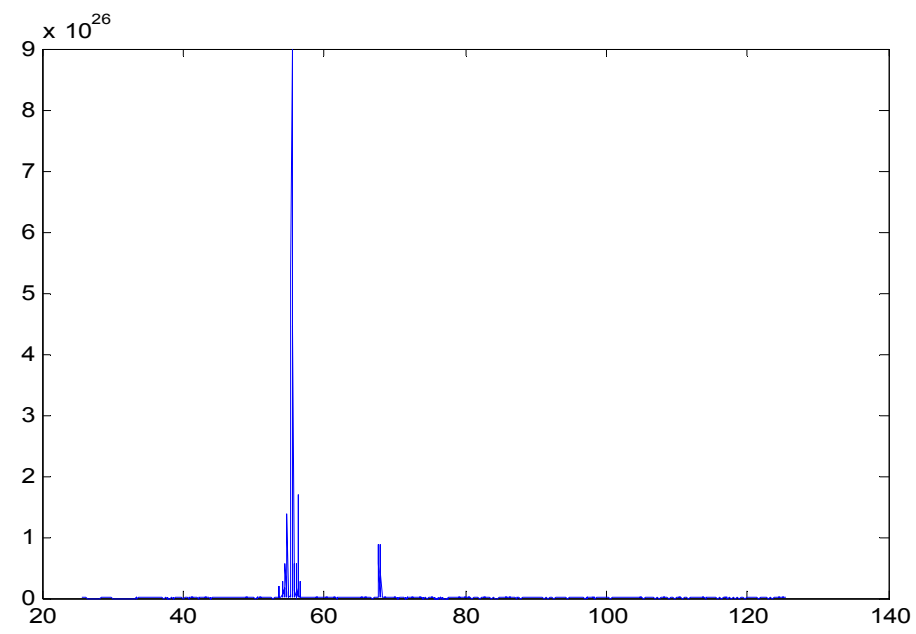

Figure 10. The results of the technical SSP (Geometric mean)

c-Echo transverse waves on

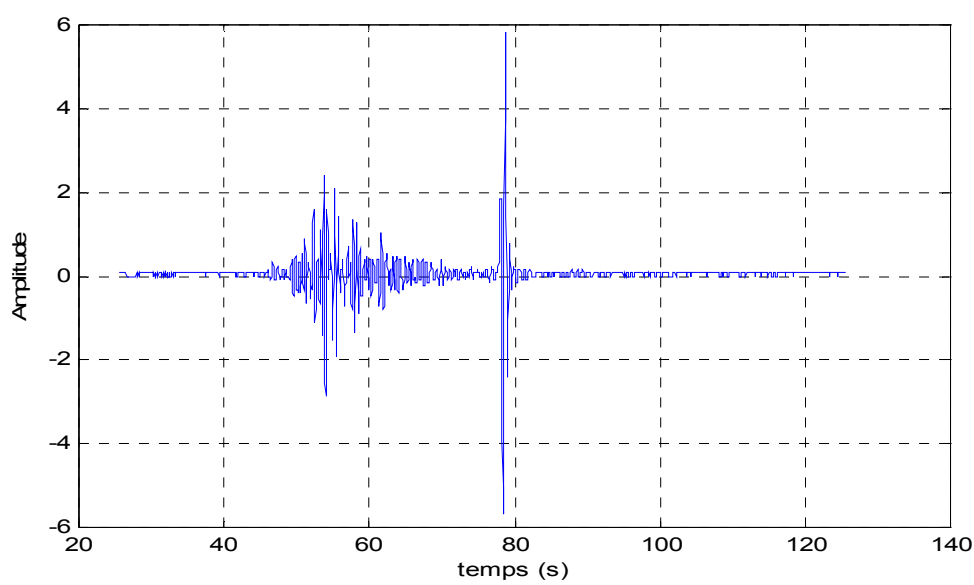

Figure 11. transverse waves

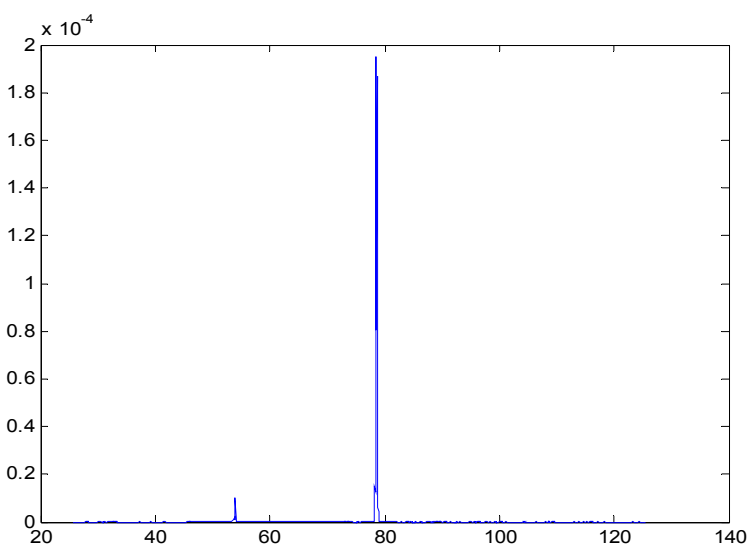

Figure 12. The results of the technical SSP (Geometric mean)

As you can see, the signals start in this first experience is clearing (widely reported). The purpose of this experiment is to demonstrate the power of technology SSP. Based on these results, it is clear that the algorithm of the geometric mean (geometric mean) gives more pronounced echoes, and therefore allows more accurate determination of the time. This will be of paramount importance when echoes are drowned

in

the

noise.

One can easily isolate the peak amplitude, and determine the time of flight. It was found the following results:

- $\mathrm{T} 1=50 \mathrm{us}$ (is the flight time for reflection of the front). - $64=\mathrm{tL}$ us (is the flight time for longitudinal waves).

$-75=\mathrm{tT}$ us (is the flight time for cross-waves).

\section{The wavelet transformation}

In this part, we applied wavelet transform and Fourier transform signals on the same treaties previously: 
- Echo of the front

- Echo longitudinal waves

- Echo transverse wave

The purpose of this technique is the same as that of SSP. Is to detect and locate the exact location of the echo and noise suppression (if any).

We also note the appearance of vertical lines (number of samples 500-1000). These lines represent the noise from the front. You can use the two precedent figures to display the energy level of the signal in the time-frequency, where the amplitude is given by the value of the level of gray color.

\section{Intercorrelation}

To elucidate the delay that may exist between two echoes. we superimposed on the figure 8 echoes emanating from the front of the sample, and the echo from the reflector. Figure 13 shows the intercorrelation signals between the front and the reflector. The delay is given by the abscissa of the maximum of the cross between two echoes. The abscissa is the number of samples (1180) which represents the difference between the echo of the reflector (number of sample $=1880$ ) and the echo of the front (number of sample $=695$ ).

-The number of sample 1880 corresponds to a time $\mathrm{t}=107$ $\mu$ s (echo reflector).

- The number of sample 685 corresponds to a time $\mathrm{t}=45 \mu \mathrm{s}$ (the echo of the front).

- The number of sample 1185 corresponds to a time $\mathrm{t}=$ $106-53=48 \mu$ s (the delay between the echo of the reflector and the echo of the front). See figures 13 and 14

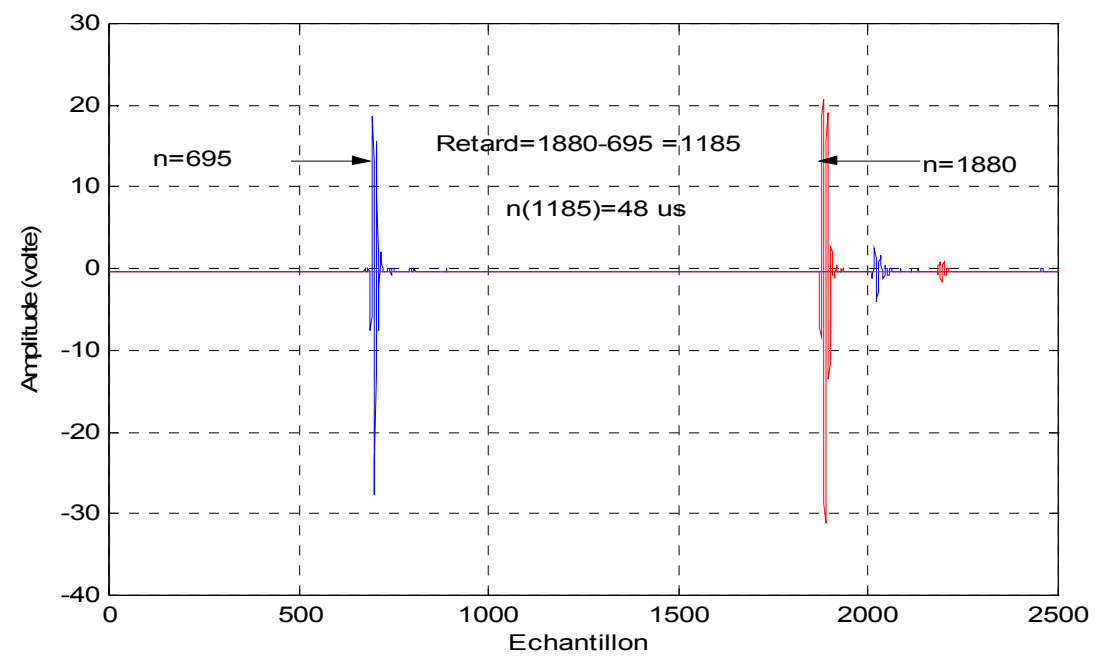

Figure 13. Two overlapping echoes (echo of the front and depth of the reflector)

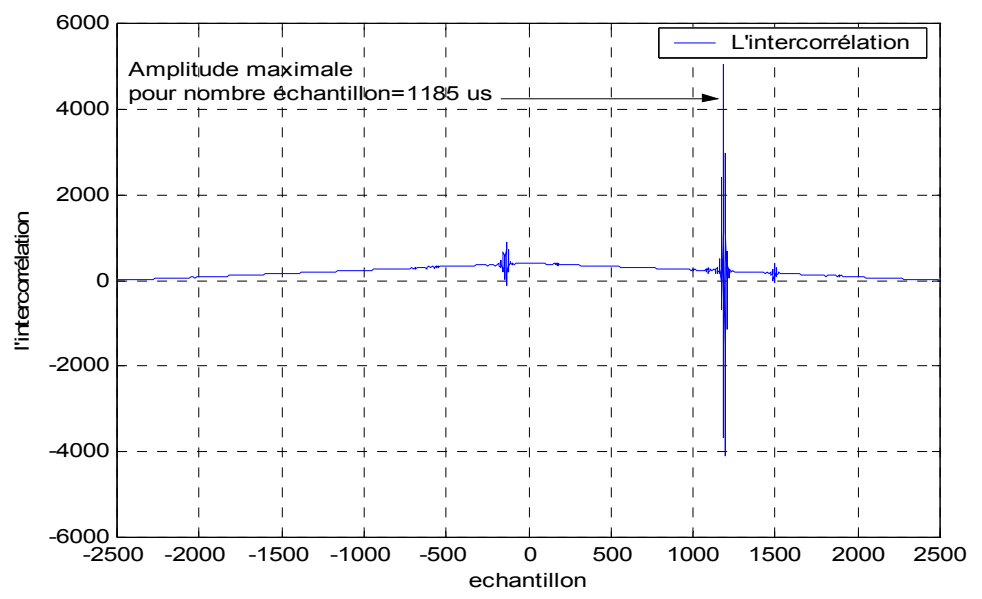

Figure. 14. - Echo longitudinal waves 


\section{Hilbert transformation:}

It can also calculate the delay time by using the Hilbert transform (Fig. 15). The delay is given by the abscissa of the maximum of the Hilbert transform. The value of the delay by the Hilbert transform is equal to 48 $\mu$ s (sample number 1185). It has exactly the same as that found by the previous method.

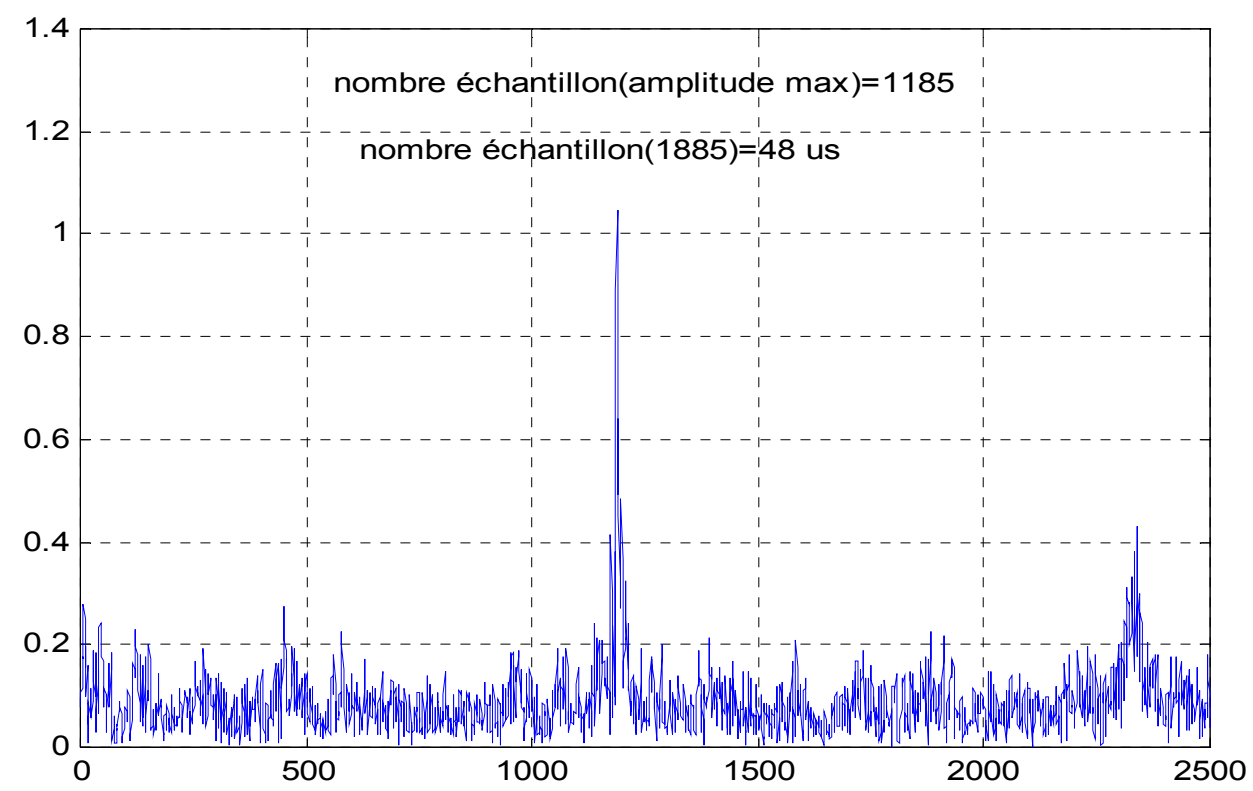

Figure 15. Hilbert presentation

\section{CONCLUSION}

In the internal structure of matter. We have presented an overview of a technique known as signal processing Split spectrum processing. This technique is based on the diversity of frequencies. Once the signal was decomposed into several bands, they were then recombined using algorithms recombination (geometric mean), which determined the location of these frequencies and the origin of echoes. With the technique Split Spectrum Processing, we got to reduce noise in granular materials (mortar and concrete). However their algorithms recombination are sensitive to filter parameters, namely the range of frequency, increasing the frequency and bandwidth of each filter. We also used the wavelet transformation to eliminate noise and echoes locate temporarily in the samples studied. As we have demonstrated, this technique has the advantage of working with bands that leads to the separation of noise.

\section{REFERENCES}

[1] A.Khireddine, K. Benmahammed, «Apllication of intelligent matching to automatic speech recognition»-ISH99 (international symposium on high voltage),.IEE conference publication, vol. 4, pp.270-P6, code ISBN.0852967195, London UK, 1999.

[2] R. Chellapa, A. Sawchuk, «Digital image processing and analysis ». Vol 1, IEEE Computer Society, 1985.

[3] A.Khireddine, K.Madi, "Global appoach of correction of geometrical satellite images ", the fourth physical congress , USTHB, vol.2, pp. 55-59, 21-23 november 2000, Alger.
[4] A.Khireddine, D. Chikouche, «Optimization by filter algorithm synthesis», AMSE, CSS'95 international conference, , vol.1, pp 9-14, Rabat, Morroco, 1995.

[5] A. Khireddine, K. Benmahammed, « application of numerical filtering for parallel treatment », CIMASI 2000 international conference, university of Rabat, vol 2, pp 132-139, Morocco, 2000

[6] A. Khireddine, H. aziri, «Application of FIR filter for restored images » IMPAC'02 conference, pp50, vol.1., code ISSN 3112-4059, IAP, Boumerdes, , Algeria, 2002

[7] A. Khireddine, K. Benmahammed, «Simulation and reconstitution of the image of a leakage currents », Journées internationales sur les sciences de la marine, Vol.2, pp321-327, Tamentfoust, Algiers, 1999

[8] A.Khireddine, K. Madi , " Geometrical Correction of satellite images by analytical aproach » IMPAC'00 conference, , vol.1, pp65-72,, code ISSN 1112-3052, Boumerdes university, Algeria, 2000.

[9] Zeng, B., Neuvo, Y., Optimal Parallel Stack Filtering under the Mean Absolute Error Criterion,IP(3), No. 3, May 1994, pp. 324-327.

[10] Links, J.M., Prince, J.L., Gupta, S.N., A vector Wiener filter for dual-radionuclide imaging, MedImg(15), No. 5, October 1996, pp. 700-709.

[11] Concetti, A., Jetto, L., Two-Dimensional Recursive Filtering Algorithm with Edge-Preserving Properties and Reduced Numerical Complexity,CirSysSignal(44), No. 7, July 1997, pp. 587-591.

[12] Wang, X., Two-Dimensional Bayesian Estimator for Image Filtering, IP(8), No. 7, July 1999, pp. 993-996.

[13] Towghi, N.[Nasser], Javidi, B.[Bahram], LP-Norm optimum filters for image recognition. Part I. Algorithms, JOSA-A(16), No. 8, August 1999, pp. 1928-1935.

[14] Gharieb, R.R., Cumulant-based LP method for two-dimensional spectral estimation, VISP(146), No. 6, December 1999, pp. 307-315.

[15] Knudsen, K.S., Bruton, L.T., Mixed domain filtering of multidimensional signals, CirSysVideo(1), No. 3, September 1999, pp. 260-268.

[16] Gharieb, R.R., Cumulant-based LP method for two-dimensional spectral estimation,VISP(146), No. 6, December 1999, pp. 307.

[17] Pokric, B., Allinson, N.M., Bergström, E.T., Goodall, D.M., Combining linear filtering and radial basis function networks for accurate profile recoverVISP(146), No. 6, December 1999, pp. 297.

[18] Kumar, B.V.K.V., Mahalanobis, A., Takessian, A., Optimal Tradeoff Circular Harmonic Function Correlation Filter Methods Providing Controlled In-Plane Rotation Response,IP(9), No. 6, June 2000, pp. 1025-1034. 
[19] Khan, I.R., Ohba, R., Hozumi, N., Noise robustness of nonlinear filters for image recognition, JOSA-A(18), No. 9, September 2001, pp. 2054-2071. 